TRANSACTIONS OF THE

AMERICAN MATHEMATICAL SOCIETY

Volume 360, Number 8, August 2008, Pages 4189-4210

S 0002-9947(08)04452-8

Article electronically published on March 12, 2008

\title{
RIGHT-ANGLED MOCK REFLECTION AND MOCK ARTIN GROUPS
}

\author{
RICHARD SCOTT
}

\begin{abstract}
We define a right-angled mock reflection group to be a group $G$ acting combinatorially on a $\mathrm{CAT}(0)$ cubical complex such that the action is simply-transitive on the vertex set and all edge-stabilizers are $\mathbb{Z}_{2}$. We give a combinatorial characterization of these groups in terms of graphs with local involutions. Any such graph $\Gamma$ not only determines a mock reflection group, but it also determines a right-angled mock Artin group. Both classes of groups generalize the corresponding classes of right-angled Coxeter and Artin groups. We conclude by showing that the standard construction of a finite $K(\pi, 1)$ space for right-angled Artin groups generalizes to these mock Artin groups.
\end{abstract}

\section{INTRODUCTION}

A right-angled Coxeter group $W$ is a group defined by a presentation in which every generator is an involution and the relations consist of commutation relations between pairs of generators. Given such a group $W$, there is a natural CAT(0) cubical complex $\Sigma$ (the so-called Davis complex) on which $W$ acts isometrically. The action has the further property that it is simply transitive on the vertices of $\Sigma$ and all edge stabilizers are isomorphic to $\mathbb{Z}_{2}$. In $[5$, it was observed that the universal covers of certain symmetric blow-ups of real reflection arrangements have group actions with these same properties. Generalizing both classes of groups we define a right-angled mock reflection group to be any group that acts isometrically on a connected $\operatorname{CAT}(0)$ cubical complex such that the action restricts to a simply transitive action on the vertex set and has $\mathbb{Z}_{2}$ edge stabilizers. The main result of this paper (Theorem 3.10) is to give a combinatorial characterization of right-angled mock reflection groups analogous to (and extending) the standard presentation for right-angled Coxeter groups.

The presentation data for a right-angled Coxeter group can be encoded in a graph whose vertices correspond to the generators and whose edges correspond to pairs of commuting generators. This graph appears in the Davis complex as the 1-skeleton of the link of a vertex. For a right-angled mock reflection group $G$ acting on a cubical complex $X$, we obtain a similar presentation encoded by a graph with local involutions. This graph is the 1-skeleton of the link of a vertex in $X$, and the local involutions keep track of "parallel translation" along paths in the 1-skeleton of $X$ (these involutions are all trivial in the case of a right-angled Coxeter group acting on the Davis complex). We show in Section 2 that this graph with involutions must

Received by the editors June 26, 2006.

2000 Mathematics Subject Classification. Primary 20Fxx.

(C)2008 American Mathematical Society Reverts to public domain 28 years from publication 
satisfy a certain "4-periodicity" condition as well as a "no holonomy" condition, and we call such a graph a mock reflection system.

Conversely, any mock reflection system $\Gamma$ determines, in a natural way, a presentation for a group $W(\Gamma)$. If all the local involutions are trivial, this group (and its presentation) is precisely the right-angled Coxeter group corresponding to the graph $\Gamma$, hence its action on the Davis complex makes it a mock reflection group. More generally we have the following.

Theorem 1. $G$ is a right-angled mock reflection group if and only if $G \cong W(\Gamma)$ for some mock reflection system $\Gamma$.

A key step in the proof of this theorem is showing that for any mock reflection system $\Gamma$, the generators of the group $W(\Gamma)$ are distinct. This follows from a simple isoperimetric inequality which we give in Theorem 3.8. Having distinct generators allows one to attach "missing" cells to the Cayley graph to obtain a CAT(0) cubical complex $\Sigma(\Gamma)$ on which $W(\Gamma)$ acts. Since this action generalizes the action of a right-angled Coxeter group on its Davis complex, we call $\Sigma(\Gamma)$ the Davis complex associated to the mock reflection group $W(\Gamma)$.

The last section of the paper describes the mock Artin group associated to a right-angled mock reflection group. In the case of an ordinary Coxeter group $W$, there is a cell complex $Y$ called the "Salvetti complex" which is related to the Davis complex. The Coxeter group acts freely on the Salvetti complex, and one manifestation of the Artin group corresponding to $W$ is that it is the fundamental group of the quotient $Y / W$. In [2] it is shown that in the right-angled setting $Y$ is nonpositively curved, a consequence of which is that the universal cover is contractible. This implies that $Y / W$ is a $K(\pi, 1)$ complex for the Artin group.

In the case of a mock reflection group $W(\Gamma)$, the $\mathrm{CAT}(0)$ complex $\Sigma(\Gamma)$ on which it acts can also be used to construct a space $Y(\Gamma)$ analogous to the Salvetti complex. Again, $W(\Gamma)$ acts freely, and the fundamental group $A(\Gamma)$ of the quotient space has a presentation given entirely in terms of the graph $\Gamma$. (As in the Coxeter group setting, the mock reflection group $W(\Gamma)$ is obtained by adding the involution relations to the presentation for $A(\Gamma)$.) We call the group $A(\Gamma)$ the mock Artin group corresponding to the mock reflection system $\Gamma$, and extend the proof in [2] to obtain the following.

Theorem 2. The group $W=W(\Gamma)$ acts freely on $Y(\Gamma)$ and the quotient space is a finite $K(\pi, 1)$ for $A(\Gamma)$.

\section{Right-ANGLED MOCK REFLECTION GROUPS}

Preliminaries. A right-angled Coxeter group (or right-angled reflection group) is a group $W$ that has a presentation of the form

$$
W=\left\langle S \mid(s t)^{m(s, t)}=1\right\rangle
$$

where $S$ is a set and $m: S \times S \rightarrow\{1,2, \infty\}$ satisfies $m(s, s)=1$ and $m(s, t)=$ $m(t, s) \in\{2, \infty\}$ otherwise. In particular, $W$ is generated by involutions, and any pair of distinct elements either commutes or has no specified relation.

Given a right-angled Coxeter group $(W, S)$, the Cayley 2-complex of the presentation can be completed to a cubical complex $\Sigma$, called the Davis complex ([3]). The complex $\Sigma$ has a natural piecewise-Euclidean metric with nonpositive curvature, and since the 2-skeleton of $\Sigma$ is a Cayley complex, $\Sigma$ is simply-connected. 
It follows that $\Sigma$ is a $\mathrm{CAT}(0)$ cubical complex (we refer the reader to 1 ] for details about CAT(0) complexes). Moreover, the action of $W$ on the Cayley complex extends to an isometric action on $\Sigma$. Since the action on the Cayley complex is simply-transitive on the vertex set and has $\mathbb{Z}_{2}$ edge stabilizers, the action on $\Sigma$ also has these properties.

Remark 2.1. Nonpositive curvature in the context of cubical complexes takes the following purely combinatorial form. Given a simplicial complex $L$, we say that $L$ is a flag complex if every collection of pairwise adjacent vertices spans a simplex in $L$. In other words, if the 1-skeleton of a simplex is in $L$, then so is the entire simplex. Now if $X$ is a cubical complex, then the link of every vertex is a simplicial complex. A well-known result of Gromov's states that a cubical complex $X$ is nonpositively-curved if and only if the link of every vertex is a flag complex.

Another class of groups that is formally similar to right-angled Coxeter groups was studied in [5]. These groups arise as automorphism groups of certain tilings obtained by taking the universal covers of symmetric blow-ups of real hyperplane arrangements. For the sake of brevity, we omit the details of this construction and refer the reader to [5]. We will, however, give a brief description of the group that arises in one prototypical case.

Let $M_{n}$ be the $(n-2)$-dimensional manifold obtained by taking the so-called minimal blow-up of the braid arrangement in $\mathbb{R} P^{n-2}$. The symmetric group $S_{n}$ acts on the braid arrangement and also on $M_{n}$, and we let $J_{n}$ be the lifted group acting on the universal cover $\tilde{M}_{n}$. The collection of hyperplanes in $\mathbb{R} P^{n-2}$ induces a $\left(S_{n^{-}}\right.$ invariant) regular cell decomposition of $M_{n}$ whose dual cellulation is a nonpositively curved cubical complex (Theorem 4.3.1 in [4]). The universal cover $\tilde{M}_{n}$, being simply connected and nonpositively curved, is a CAT(0) cubical complex on which $J_{n}$ acts combinatorially. This action is simply transitive on the vertices of $\tilde{M}_{n}$ and the edge stabilizers are all $\mathbb{Z}_{2}$. The group $J_{n}$ has a presentation with generators $s_{i j}$ for $1 \leq i<j \leq n$, and relations

(1) $s_{i j}^{2}=1$,

(2) $s_{i j} s_{k l}=s_{k l} s_{i j}$ for all $i<j<k<l$, and

(3) $s_{i j} s_{k l}=s_{k^{\prime} l^{\prime}} s_{i j}$ where $i<k<l<j, k^{\prime}=i+j-l$, and $l^{\prime}=i+j-k$

(this is a special case of Theorem 4.7.2 in [5].) Notice that, as for right-angled Coxeter groups, $J_{n}$ is generated by involutions, and all (non-involution) relations have length 4 .

Remark 2.2. By results of Kapranov 7 and [8, the manifold $M_{n}$ is homeomorphic to both (1) a certain compactification of the quotient space $\mathcal{C}_{n+1} / P G L_{2}$ where $\mathcal{C}_{n+1}$ denotes the set of generic configurations of $(n+1)$-points in $\mathbb{R} P^{1}$ and (2) the real points of the Grothendieck-Knudsen-Deligne-Mumford moduli space of stable curves of genus 0 with $(n+1)$-marked points. Since $\tilde{M}_{n}$ is $\operatorname{CAT}(0)$, it is contractible, so $M_{n}$ is a $K(\pi, 1)$-space. The fundamental group $\pi=\pi_{1}(M)$ is a finite index subgroup of $J_{n}$. Because of this connection to stable real curves with marked points, the group $J_{n}$ is sometimes called the cactus group and $\pi_{1}(M)$ the pure cactus group. The cohomology of $M$ (and, hence, of the pure cactus group) has recently been computed in 6 .

The two types of groups described above, right-angled Coxeter groups and groups acting on universal covers of blow-ups, motivate the following definition. 
Definition 2.3. A group $G$ is a right-angled mock reflection group (RAMRG) if it acts isometrically on a connected CAT(0)-cubical complex $X$ such that the action is simply-transitive on the 0-skeleton, and the stabilizer of every edge is isomorphic to $\mathbb{Z}_{2}$.

Generators and relations. Suppose $G$ is a RAMRG with corresponding cubical complex $X$. By fixing a basepoint $x_{0} \in X^{(0)}$, we obtain a bijection $G \rightarrow X^{(0)}$ given by $a \mapsto a \cdot x_{0}$. Let $V$ denote the set of vertices adjacent to $x_{0}$, and for each $v \in V$, let $s_{v} \in G$ denote the element of $G$ such that $s_{v} \cdot x_{0}=v$. This element $s_{v}$ is unique (simple transitivity) and has order 2 (it stabilizes the edge $\left\{x_{0}, v\right\}$ ).

Proposition 2.1. G is generated by the set of involutions $\left\{s_{v} \mid v \in V\right\}$.

Proof. Given $g \in G$, let $x=a \cdot x_{0}$, and let $x_{0}, x_{1}, \ldots, x_{n}=x$ be a path (sequence of adjacent vertices) joining $x_{0}$ to $x$. If $n=1$, then $x \in V$, so $a=s_{x}$. Otherwise, we can assume by induction that $x_{n-1}=b \cdot x_{0}$ where $b$ can be expressed as a product of the $s_{v}$ 's. Let $c$ be the (unique) element of $G$ such that $x=c \cdot x_{n-1}$. Then $b^{-1} c b$ maps $x_{0}$ to an adjacent vertex, say $v^{\prime}$, hence $b^{-1} c b=s_{v^{\prime}}$. It follows that $x=a \cdot x_{0}=c b \cdot x_{0}=b s_{v^{\prime}} \cdot x_{0}$, so $a=b s_{v^{\prime}}$ by simple-transitivity.

It follows from this proposition that the 1-skeleton of $X$ can be identified with the Cayley graph of $G$ with respect to the generating set $\left\{s_{v} \mid v \in V\right\}$. In other words, if we identify $G$ with the 0 -skeleton of $X$ by the map $a \mapsto a \cdot x_{0}$, then two vertices $a \cdot x_{0}$ and $a^{\prime} \cdot x_{0}$ are joined by an edge if and only if $a^{\prime}=a s_{v}$ for some generator $s_{v}$ (since $s_{v}$ is an involution, this edge is undirected). In particular, each edge in $X$ acquires a unique label from the set $V$ : the edge $\left\{a, a^{\prime}\right\}$ is labeled by the unique $v$ such that $a^{\prime}=a s_{v}$. Since the group acts on the left, this labeling is $G$-invariant.

Given 2 vertices $u$ and $v$ in $V$, we say that $u$ and $v$ span a square at $x_{0}$ if there exists a vertex $w$ in $X$ and a 2-cell (i.e., a square) $R$ such that the vertices of $R$ are $u, x_{0}, v, w$ (in order). Since $u=s_{u} \cdot x_{0}$ and $v=s_{v} \cdot x_{0}$, it follows (as in the proof above) that there exists a (unique) generator $s_{u^{\prime}}$ such that $w=s_{u^{\prime}} \cdot v=s_{v} s_{u^{\prime}} \cdot x_{0}$ (Figure 11). The element $u^{\prime} \in V$ actually depends on both $u$ and $v$, so we adopt the notation $u^{\prime}=j_{v}(u)$. Notice that, in general, since the edge labels are $G$-invariant,

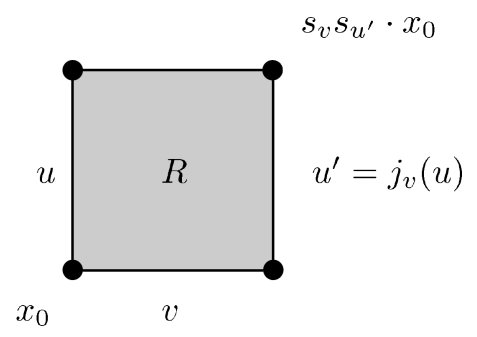

FiguRE 1

$j_{v}(u)$ can be determined from the labels on any square that has labels $u$ and $v$ on consecutive edges (whether these edges meet at $x_{0}$ or not). Namely, by traveling the boundary of the square starting at the edge labeled $u$, then continuing to $v$, the next edge must have the label $j_{v}(u)$. As a consequence, we have the following. 
Proposition 2.2. Let $V_{v}$ denote the set of vertices $u \in V$ such that $u$ and $v$ span a square at $x_{0}$. Then

(i) $u \mapsto j_{v}(u)$ defines an involution $j_{v}: V_{v} \rightarrow V_{v}$.

(ii) The sequence $v_{0}, v_{1}, \ldots$ defined by $v_{0}=u, v_{1}=v$, and $v_{k+1}=j_{v_{k}}\left(v_{k-1}\right)$ for $k \geq 1$ is periodic with period 4 .

Proof. Let $R$ be a square in $X$ with consecutive edge labels $u$ and $v$ as in Figure 2 , Since $v$ and $j_{v}(u)$ are also consecutive labels in the square $R$, we have $j_{v}(u) \in V_{v}$. Traveling the boundary of $R$ in the opposite direction starting at $j_{v}(u)$, we see that $j_{v} j_{v}(u)=u$, so $j_{v}$ is an involution. This proves (i). For (ii), just observe that the sequence $v_{0}, v_{1}, v_{2}, \ldots$ is the sequence of edge-labels as we travel around the square $R$.

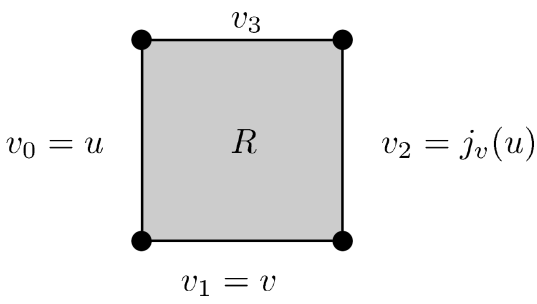

FiguRE 2

The involutions $j_{v}$ also satisfy a "trivial holonomy" condition, which we now describe. Let $u, v, w \in V$ be three vertices such that any pair of them spans a square at $x_{0}$. Then in the link of $x_{0}$, the vertices $u, v, w$ are pairwise joined by edges. Since $X$ is nonpositively curved, it follows by Gromov's condition that there must be a 3 -cube at $x_{0}$ whose link "fills in" this triangle. That is, there exists a 3 -cube in $X$ with edges $\left\{x_{0}, u\right\},\left\{x_{0}, v\right\}$, and $\left\{x_{0}, w\right\}$. In this case, we say that $u, v, w$ span a cube at $x_{0}$.

Proposition 2.3. Suppose $u, v, w$ span a cube at $x_{0}$, and let $v_{0}, v_{1}, \ldots$ be the sequence defined in Proposition 2.2 .

(i) Then the triple $v, j_{v}(u), j_{v}(w)$ spans a cube at $x_{0}$.

(ii) The sequence $w_{0}, w_{1}, \ldots$ defined by $w_{0}=w$ and $w_{k}=j_{v_{k}}\left(w_{k-1}\right)$ is also periodic with period 4.

Proof. Let $Q$ be the 3-cube at $x_{0}$ spanned by $u, v, w$ as in Figure 3 , For (i) note that each pair from $v, j_{v}(u), j_{v}(w)$ occurs as consecutive labels in a square face of $Q$, hence the triple $v, j_{v}(u), j_{v}(w)$ must also span a cube. The second part (ii) follows from the fact that traveling around the bottom square, the labels on the vertical edges perpendicular to each vertex form the sequence $w_{0}, w_{1}, \ldots$.

The first proposition above determines some additional relations for the group $G$. Namely, given a pair of vertices $u, v \in V$ that span a square at $x_{0}$, let $u, v, u^{\prime}, v^{\prime}$ be the edge labels on this square. Then the group element $s_{u} s_{v} s_{u^{\prime}} s_{v^{\prime}}$ maps $x_{0}$ to itself, so by simple transitivity, it must be the identity. It turns out that, together with the involution relations, these relations suffice. 


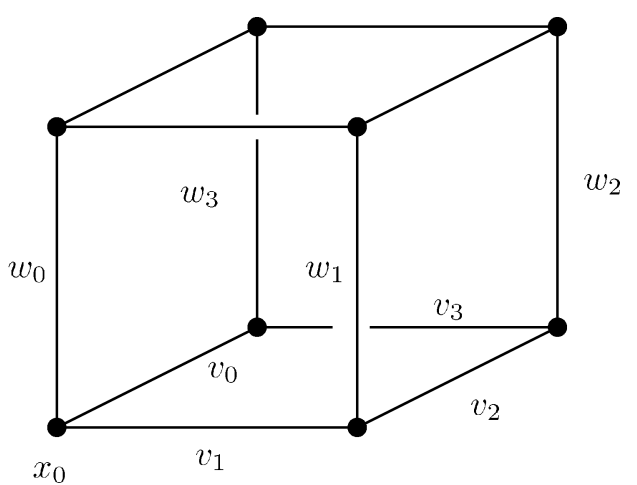

Figure 3

Proposition 2.4. The group $G$ is determined by the relations

(i) $s_{v} s_{v}=1$ for every $v \in V$, and

(ii) $s_{v} s_{u} s_{v^{\prime}} s_{u^{\prime}}=1$ whenever $u$ and $v$ span a square at $x_{0}$ (and $u^{\prime}=j_{v}(u)$ and $\left.v^{\prime}=j_{u}(v)\right)$.

Proof. If we identify $X^{(1)}$ with the Cayley graph of $G$, then to construct the Cayley 2 complex associated to this presentation, we glue in a square whenever we have 4 vertices of the form $a, a s_{v}, a s_{v} s_{u}, a s_{v} s_{u} s_{v^{\prime}}$ (up to cyclic permutations and inversions) where $u$ and $v$ span a square at $x_{0}(=1 \in G)$. It follows that $X^{(2)}$ can be identified with the Cayley complex of the group defined by the generators $\left\{s_{v}\right\}$ and the relations (i) and (ii). (Strictly speaking, one must first reduce the set of relations so there are no cyclic conjugates or inverses among the list.) Any relation among the generators of $G$ would determine a loop in $X^{(2)}$ and hence a loop in this Cayley complex. But any loop in a Cayley complex is a composition of loops corresponding to the relators (and their conjugates). It follows that any such relation is a consequence of the relations listed above.

\section{Mock RefleCtion Systems}

4-periodic graphs and holonomy. Let $\Gamma$ be a simple graph with vertex set $V$. For each $v \in V$, let $\Gamma_{v}$ denote the induced subgraph on the neighbors of $v$, and let $j_{v}: \Gamma_{v} \rightarrow \Gamma_{v}$ be an automorphism such that $\left(j_{v}\right)^{2}=$ Id. We illustrate this data by drawing the graph $\Gamma$ with edges paired up at each vertex; that is, we connect two edges $\{u, v\}$ and $\left\{v, u^{\prime}\right\}$ by an arc at the vertex $v$ whenever $j_{v}(u)=u^{\prime}$. For example, in Figure 4, the local involution $j_{a}$ fixes $e$ and $c$ and interchanges $b$ and $d$. We call the pair $\left(\Gamma,\left\{j_{v}\right\}\right)$ (or just $\Gamma$, if the involutions are implied) a graph with local involutions.

Let $\Gamma$ be a graph with local involutions. Then any pair $u, v$ of adjacent vertices determines a sequence $\tau(u, v)=v_{0}, v_{1}, \ldots$ where $v_{0}=u, v_{1}=v$, and $v_{k+1}=$ $j_{v_{k}}\left(v_{k-1}\right)$ for $k \geq 2$. We call $\tau(u, v)$ a trajectory. Since $V$ is finite, every trajectory will eventually repeat, and we say that $\Gamma$ is 4-periodic if $v_{n}=v_{n+4}$ for all $n \geq 0$. Note that for such a trajectory, $v_{0}, v_{1}, v_{2}, v_{3}, v_{0}, v_{1}, \ldots$, consecutive vertices will be distinct, but we could still have $v_{i}=v_{i+2}$ for $i$ even or $i$ odd (or both). In terms of the diagram for $\Gamma$, this means that the edges of $\Gamma$ can be partitioned into sets of 3 types: a single edge not paired at either endpoint, two edges paired only with each 


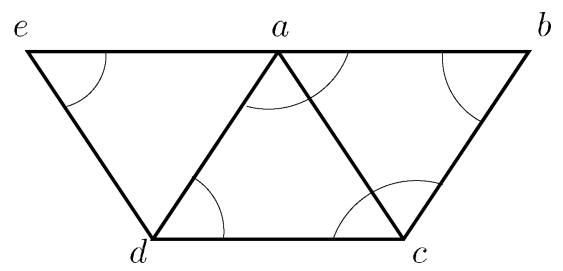

FIGURE 4

other (at a shared vertex), or 4-cycles of edges paired in cyclic order (Figure 5). We call such a grouping of edges in the partition an edge set, and an edge set with
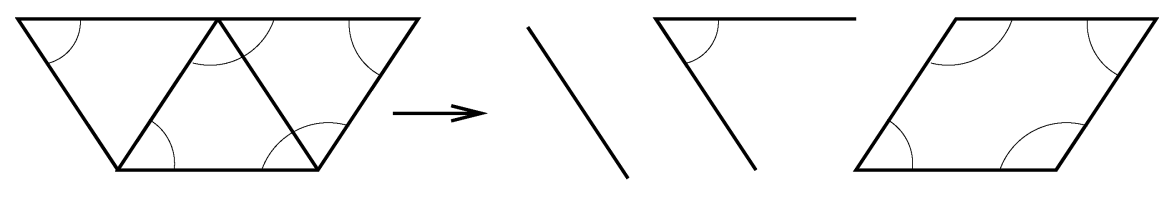

Figure 5

$k$-edges a $k$-edge set.

Given a graph $\Gamma$ with local involutions, we also have the notion of parallel transport along trajectories. Given a trajectory $\tau(u, v)=v_{0}, v_{1}, v_{2}, \ldots$, suppose $w_{0}$ is a vertex adjacent to both $v_{0}(=u)$ and $v_{1}(=v)$. Then since the involution $j_{v_{1}}$ preserves adjacency, the vertex $w_{1}=j_{v_{1}}\left(w_{0}\right)$ will be adjacent to both $v_{1}$ and $v_{2}=j_{v_{1}}\left(v_{0}\right)$. Proceeding by induction, we define $w_{k}=j_{v_{k}}\left(w_{k-1}\right)$, which will in turn be adjacent to both $v_{k}$ and $v_{k+1}$ (Figure 6).

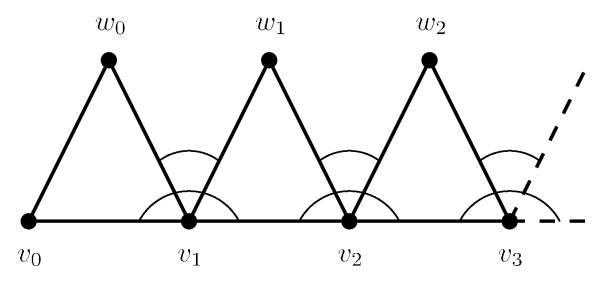

FiguRE 6

Now assume $\Gamma$ is 4-periodic, and let $\Gamma_{u v}$ denote the induced subgraph on the set of vertices adjacent to both $u$ and $v$. Then the map $w_{0} \mapsto w_{4}$ defines an automorphism $\phi_{u v}: \Gamma_{u v} \rightarrow \Gamma_{u v}$. We call this map the holonomy corresponding to the pair $(u, v)$. (Of course, there is an analogous notion of holonomy for any graph with local involutions, not just 4-periodic ones.)

Definition 3.1. Let $\Gamma$ be a graph with local involutions. Then $\Gamma$ is a (right-angled) mock reflection system if it is 4-periodic and all holonomy maps are trivial.

The example in Figure 8 shows that a 4-periodic graph need not have trivial holonomy (the holonomy map $j_{d} \circ j_{a} \circ j_{b} \circ j_{a}$ interchanges the vertices $c$ and $d$ ). On the other hand, suppose $\Gamma$ is obtained from the action of a mock reflection group $G$ on its cubical complex $X$. In other words, $\Gamma$ is the 1 -skeleton of the link of 
$x_{0} \in X$, and for each vertex $v$ of $\Gamma, j_{v}$ is the involution defined in Section 2, Then by Proposition 2.2. $\Gamma$ is 4-periodic, and by Proposition 2.3 all holonomy maps are trivial. Hence $\Gamma$ is a mock reflection system.

The group $W(\Gamma)$. Suppose $\Gamma$ is a 4-periodic graph, and let $F(V)$ denote the free group on the vertex set $V$. For any pair $(u, v) \in V \times V$, define the element $r(u, v) \in F(V)$ by

$$
r(u, v)= \begin{cases}u^{2} & \text { if } u=v, \\ u v u^{\prime} v^{\prime} & \text { if } u \text { and } v \text { are adjacent, } u^{\prime}=j_{v}(u) \text { and } v^{\prime}=j_{u}(v), \\ 1 & \text { otherwise. }\end{cases}
$$

Let $W(\Gamma)$ be the group defined by the generators $v \in V$ and relations $r(u, v)$. That is, $W(\Gamma)=F(V) / N$ where $N$ is the normal subgroup generated by $r(u, v)$ for all $(u, v) \in V \times V$.

In light of the partition of the edges, we can eliminate many of the relations in the defining presentation for $W(\Gamma)$. Given any two edges $\{u, v\},\{w, z\}$ in an edge set the relation $r(u, v)$ can be obtained from $r(w, z)$ by cyclically permuting letters and taking inverses (since all generators are involutions). Hence, it suffices to specify, in addition to the relation $\left(s_{v}\right)^{2}$ for each vertex, exactly one relation for each edge set.

Example 3.2. If $\Gamma$ is a triangle, there are exactly two possibilities. These are both mock reflection systems and are shown in Figure 7 . The corresponding presentations for $W(\Gamma)$ are

$$
\left\langle a, b, c \mid a^{2}=b^{2}=c^{2}=1,(a b)^{2}=(b c)^{2}=(a c)^{2}=1\right\rangle
$$

and

$$
\left\langle a, b, c \mid a^{2}=b^{2}=c^{2}=1, a b a c=1,(b c)^{2}=1\right\rangle,
$$

which are the groups $\left(\mathbb{Z}_{2}\right)^{3}$ (a right-angled Coxeter group) and $D_{4}$ (the dihedral group of order 8), respectively.
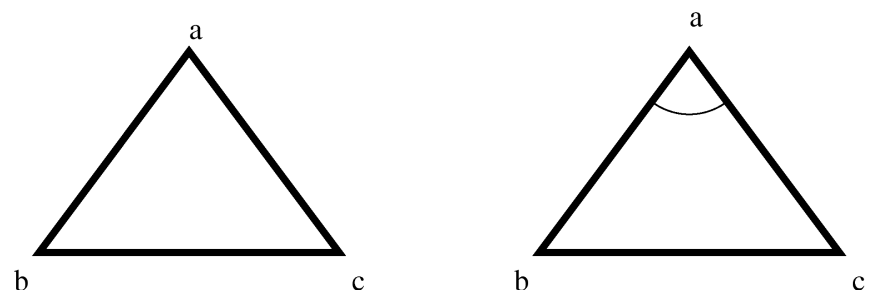

FiguRE 7

Example 3.3. Let $\Gamma$ be the graph $K_{4}$ with local involutions defined as in Figure 8 , In this case $\Gamma$ is not a mock reflection system since it has nontrivial holonomy. Nonetheless, it determines a group $W(\Gamma)$ by the presentation

$$
\left\langle a, b, c, d \mid a^{2}=b^{2}=c^{2}=d^{2}=1, a b a d=b d b c=c a c d=1\right\rangle .
$$

By eliminating $b$ and $c$ from the presentation we obtain

$$
\left\langle a, d \mid a^{2}=d^{2}=1,(a d)^{7}=1\right\rangle ;
$$

thus, the group $W(\Gamma)$ is the dihedral group $D_{7}$ of order 14 . 


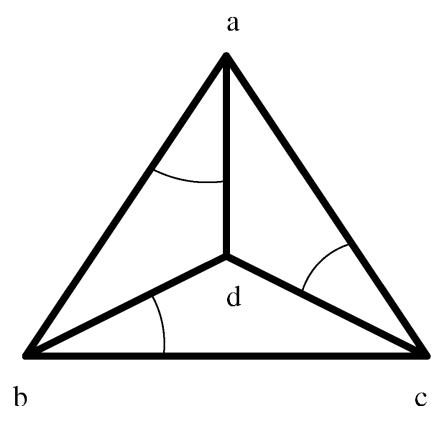

FigURE 8

Suppose $W$ is any right-angled Coxeter group, and let $\Gamma$ be the graph with one vertex for each generator and an edge joining two vertices whenever the corresponding generators commute. For each vertex $v$, let $j_{v}$ be the trivial local involution. Then $\Gamma$ is 4-periodic (in fact, it is 2-periodic), and the group $W(\Gamma)$ is precisely $W$.

More generally, suppose $G$ is a RAMRG acting on its cubical complex $X$. Let $\Gamma$ be the 1-skeleton of the link of $x_{0} \in X$, and for each vertex $v$ of $\Gamma$, let $j_{v}$ be the involution defined in Section 2, Then as mentioned above, $\Gamma$ is a mock reflection system, and by Proposition $2.4 G$ is isomorphic to the group $W(\Gamma)$.

Example 3.4. As mentioned in the Preliminaries in Section 2 the cactus group $J$ acting on the universal cover $\tilde{M}$ of the moduli space of genus zero curves with $(n+3)$ marked points is an example of a RAMRG. The corresponding mock reflection system $\Gamma$ is shown in Figure 9 in the first two cases $(n=1,2)$. For general $n$,
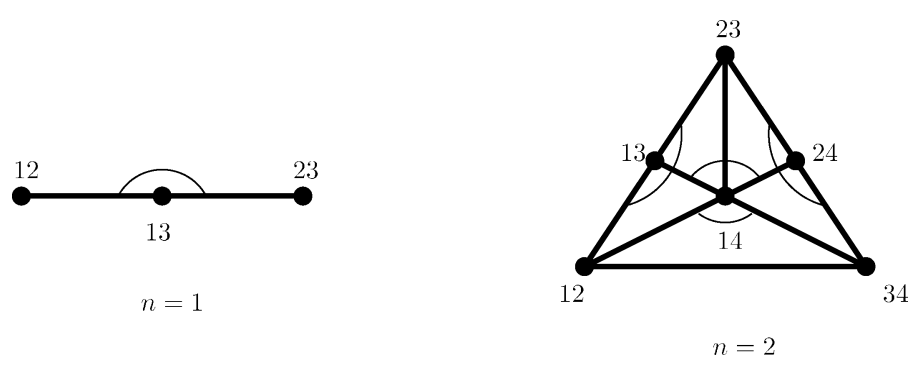

FigURE 9. Mock reflection systems for cactus groups

suppose $\Delta$ is the simplex with vertex set $\left\{s_{12}, s_{23}, \ldots, s_{n+1}{ }_{n+2}\right\}$. Let $s_{i j}$ denote the barycenter of the face $\left\{s_{i} i_{+1}, s_{i+1} i+2, \ldots s_{j-1}\right\}$. Then $\Gamma$ can be viewed as the 1-skeleton of the stellar subdivision of $\Delta$ obtained by iteratively stellating on these barycenters starting with the deepest barycenter $s_{1 n+2}$ first. The involutions $j_{s_{i j}}$ are induced by the involution in the symmetric group (acting on $\Delta$ ) that reverses the order of the interval $i, i+1, \ldots, j$. (A detailed explanation of the relationship between blow-ups of subspace arrangements and stellar subdivisions can be found in Section 3.3 of [4.)

van Kampen diagrams. Let $\Gamma$ be a mock reflection system. It turns out that 4-periodicity and trivial holonomy have nice implications for the topology of van Kampen diagrams for the group $W(\Gamma)$. This leads to an explicit isoperimetric 
inequality which we shall use to prove that the generators of $W(\Gamma)$ are distinct. First we reformulate some basic facts from combinatorial group theory in the context of groups generated by involutions.

Let $V$ be a finite set and let $F_{2}(V)$ denote the free amalgamation $*_{V} \mathbb{Z}_{2}$ with one factor for each element of $V$. Letting $v$ denote the free generator corresponding to an element $v \in V$, we say that an element $v_{1} v_{2} \cdots v_{n} \in F_{2}(V)$ is a reduced word if $v_{i} \neq v_{i+1}$ for $i=1, \ldots, n-1$ and is cyclically reduced if in addition $v_{n} \neq v_{1}$. We define the length of an element $w \in F_{2}(V)$ to be the number of generators in a reduced expression for $w$.

For any collection $R \subset F_{2}(V)$, let $N$ be the normal subgroup generated by $R$, and let $G=F_{2}(V) / N$. Any element $w \in N$ can be written in the form

$$
\prod_{i=1}^{m} w_{i} r_{i}^{ \pm 1} w_{i}^{-1}
$$

where $r_{i} \in R$ and $w_{i} \in F_{2}(V)$ (of course $w_{i}=v_{1} \cdots v_{n}$ implies $w_{i}^{-1}=v_{n} \cdots v_{1}$ ). Corresponding to any such representation for $w$ there exists a connected simplyconnected planar cell complex called the van Kampen diagram: each 1-cell in this complex is labeled by a generator, the labels around any 2-cell determine a word in $R$ (up to cyclic permutations and inversions), and the boundary cycle for the entire complex (with respect to some fixed boundary vertex) is the word $w 1$ Conversely, for any such diagram, the full boundary label determines an element of $N$.

For any given word $w \in N$, we define its area $\alpha(w)$ to be the minimal number of 2-cells needed for a van Kampen diagram for $w$. That is,

$$
\alpha(w)=\min \left\{m \mid w=\prod_{i=1}^{m} w_{i} r_{i}^{ \pm 1} w_{i}^{-1} \quad r_{i} \in R\right\} .
$$

For the groups we are interested in, the set of relators $R$ will consist only of 4letter words. In this case, any word in $N$ has a van Kampen diagram whose 2-cells are all squares. The planar dual of such a diagram can be drawn as a collection of closed smooth immersed curves such that all intersections are transverse double points with the possible exception of the single point $*$ corresponding to the exterior face of the van Kampen diagram. By cutting out a disk that separates $*$ from the van Kampen diagram, we obtain a collection of curves in the disk that are closed or start and end on the boundary circle (see Figure 10). We call such a picture a chord diagram.

Now assume $\Gamma$ is a mock reflection system with vertex set $V$. Let $\mathcal{R}$ be the set of 4-term relations (in $F_{2}(V)$ ) corresponding to trajectories in $\Gamma$, and let $N$ be the normal subgroup generated by $\mathcal{R}$. Note that each of the relations in $\mathcal{R}$ is cyclically reduced; hence in any van Kampen diagram, the boundary cycle of a 2-cell never has consecutive repeated labels. (In particular, a pendant vertex like the one occurring in the van Kampen diagram of Figure 10 cannot occur.) We then have the following two types of "moves" for van Kampen diagrams (and their duals).

Type I move. If a diagram contains an interior vertex of degree 2, then there are 2 adjacent regions sharing consecutive edges (Figure 11). Since the labels $a, b$ on these consecutive edges determine a unique trajectory $a, b, c, d, \ldots$, the boundary

\footnotetext{
${ }^{1}$ The usual van Kampen diagram specifies labels for oriented 1-cells (see, e.g., [9]), but since all of our generators are involutions, there is an obvious reduction to unoriented diagrams.
} 


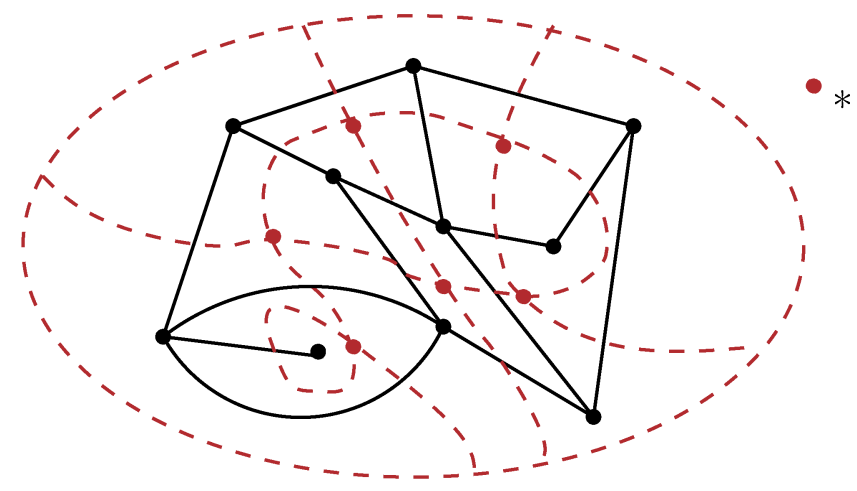

Figure 10. A 4-term van Kampen diagram and dual chord diagram

cycles are the same for both regions. It follows that the 2 squares can be reduced to just a pair of incident edges labeled $c, d$. The corresponding move for chord diagrams is also shown in Figure 11 .

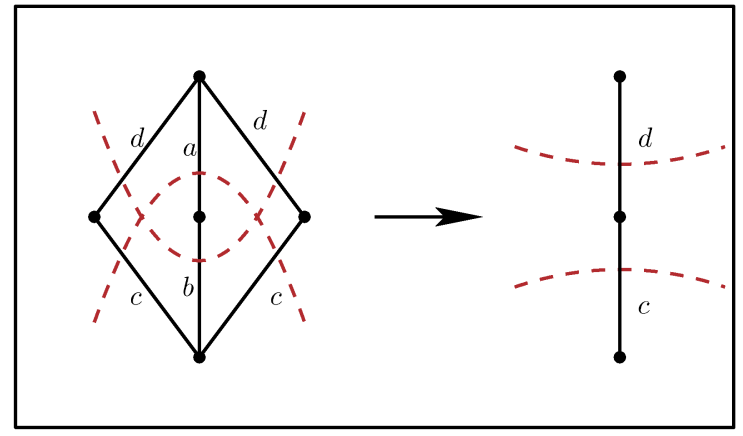

Figure 11. Type I move

Type II move. If a diagram contains an interior vertex $v$ of degree 3 , then three 2 -cells meet at $v$, and the boundary of this configuration is a 6-cycle (Figure 12). The three edges emanating from $v$ meet the 6 -cycle in alternate vertices. This configuration can be replaced with a similar diagram (preserving the outside labels) but with the three interior edges meeting the other three vertices of the boundary 6 -cycle. To see this, note that the interior labels $a, b, c$ must be different generators and the exterior labels are then given by

$$
b^{\prime}=j_{a}(b), b^{\prime \prime}=j_{c}(b), a^{\prime}=j_{b}(a), a^{\prime \prime}=j_{c}(a), c^{\prime}=j_{a}(c), c^{\prime \prime}=j_{b}(c) .
$$

The new interior labels are given by

$$
a^{\prime \prime \prime}=j_{b^{\prime \prime}}\left(a^{\prime \prime}\right)=j_{c^{\prime \prime}}\left(a^{\prime}\right), b^{\prime \prime \prime}=j_{a^{\prime \prime}}\left(b^{\prime \prime}\right)=j_{c^{\prime}}\left(b^{\prime}\right), c^{\prime \prime \prime}=j_{b^{\prime}}\left(c^{\prime}\right)=j_{a^{\prime}}\left(c^{\prime \prime}\right)
$$

where the equalities all follow from the trivial holonomy condition. We leave it to the reader to verify that the boundary cycles for the three new 2-cells are, in fact, relations in $\mathcal{R}$. Again, the dual move for chord diagrams is also shown in Figure 12 ,

Remark 3.5. In terms of the dual chord diagram, the moves described above coincide with the Reidemeister type moves obtained by Sageev [11 in perturbing a 


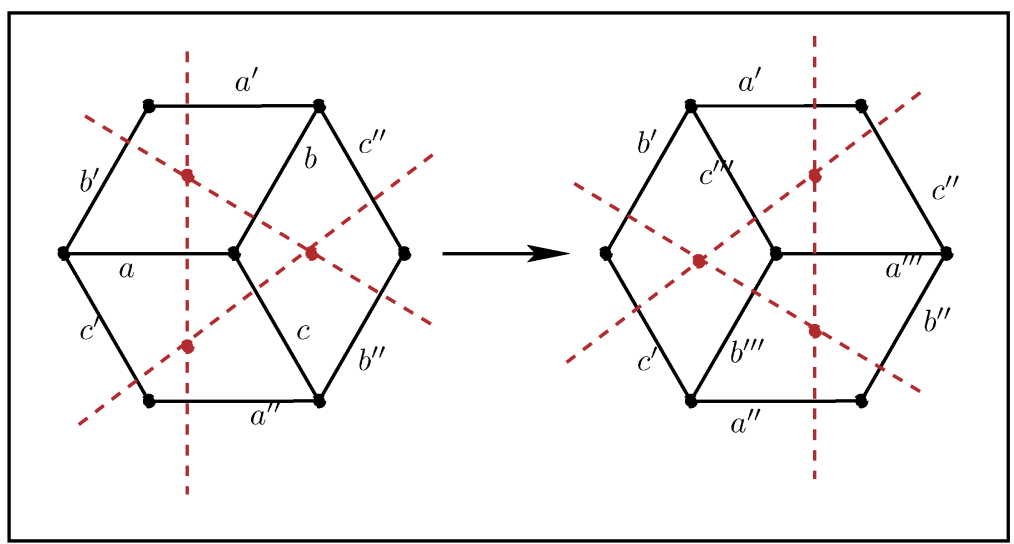

Figure 12. Type II move

contracting disk in a CAT(0) cubical complex. Here, however, we make no assumption about the existence of an ambient CAT(0) cube complex. Our only assumption is that the presentation has no holonomy.

An isoperimetric inequality. We shall now use the moves described above to deduce an isoperimetric inequality for relators in the group $W(\Gamma)$. Our arguments will be clearer if we work with chord diagrams instead of van Kampen diagrams, since for the former the moves described above have nice topological interpretations as Reidemeister moves for curves in the plane. Note that the number of 2-cells in a van Kampen diagram corresponds to the number of interior crossings or "vertices" in the dual chord diagram.

Definition 3.6. A chord diagram $D$ is taut if it satisfies all of the following conditions:

(1) None of the chords or interior curves have self-intersections.

(2) There are no interior closed curves (i.e., $D$ only contains chords meeting the boundary).

(3) No two chords intersect more than once.

Given a chord diagram $D$, let $C$ be any simple closed curve that only intersects curves in $D$ transversely. Then the region $D^{\prime}$ bounded by $C$ is also a chord diagram, which we call a subdiagram of $D$. For chord diagrams and their subdiagrams, we will often omit the boundary circle.

Our first observation is that a chord diagram that is not taut must contain at least one subdiagram $E$ having the following form. $E$ has two distinct chords $\alpha$ and $\beta$ such that

(i) $\alpha$ and $\beta$ intersect in exactly 2 points $p$ and $q$,

(ii) $\alpha$ and $\beta$ have no self-intersections, and

(iii) $E$ has no interior vertices (crossings) outside the region enclosed by $\alpha$ and $\beta$ (Figure 13).

The proof of this fact is a little tedious, but not difficult. For example, if condition (1) in Definition 3.6 fails, then there exists a self-intersecting curve $\alpha$. If this curve has a loop (Figure 14), then some other chord must cross it (if no chords cross it, 


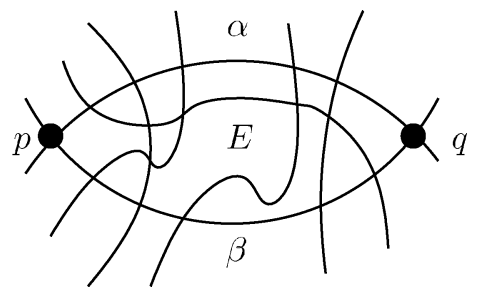

FIGURE 13

then the dual van Kampen diagram would have an interior pendant vertex which is impossible since the relators in $\mathcal{R}$ are all cyclically reduced). The crossing curve $\beta$ might self-intersect inside this loop, but by taking a minimal such loop in $D$, we can make sure it doesn't. In this case we obtain $E$ by taking the smallest subdiagram containing the region bounded by the crossing curve $\beta$ and the piece of $\alpha$ it cuts off (Figure 14, left side). If the curve $\alpha$ has no loop, then it has at least two self-intersection points $p$ and $q$, which we can take to be consecutive along one of the connecting arcs. We can then find a simply connected subregion bounded by this arc and another connecting arc. We then let $E$ be the smallest subdiagram containing this region (Figure 14, right side). The cases where conditions (2) or (3) fail are similar.
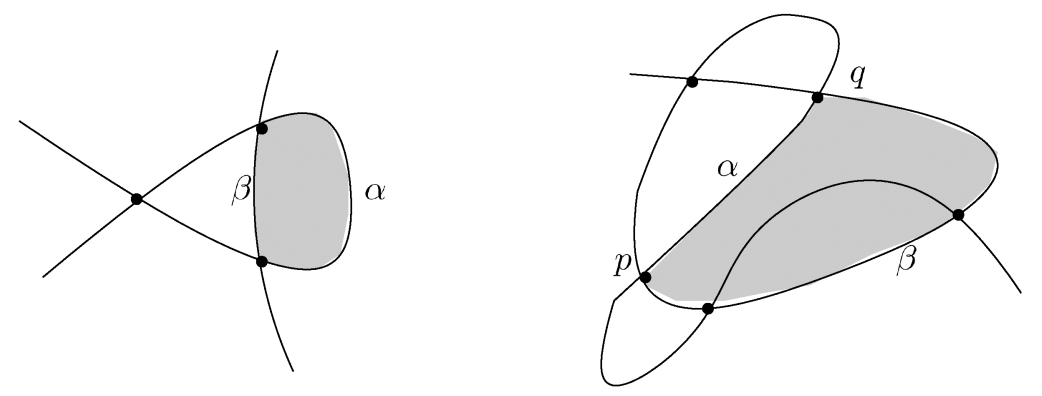

FiguRE 14

If we know that a diagram $E$ of the above type exists, then we can take a minimal one, i.e., one that has no further subdiagram of this same type. Minimality implies that $E$ has no self-intersecting curves, no closed curves, and the only two curves that intersect more than once are $\alpha$ and $\beta$ (and they intersect exactly twice). In particular, if $\gamma$ is any chord other than $\alpha$ and $\beta$, then $\gamma$ must cross $\alpha$ precisely once (and before crossing any other chord) and must cross $\beta$ exactly once (and cross no other chords after $\beta$ ). This means we can regard $E$ as a plane projection of a braid where the strands of the braid correspond to the chords other than $\alpha$ and $\beta$, no strand self-intersects, and no two strands intersect more than once. In other words, ignoring $\alpha$ and $\beta$, we obtain a "taut" braid projection (Figure 15).

The proof of the following lemma is essentially the same as the proof of Theorem 4.4 in [11]. We include it here for completeness.

Lemma 3.7. Let $D$ be a chord diagram for a word $w$ such that $D$ has the minimal number of interior crossings (i.e., $D$ has $\alpha(w)$ interior crossings). Then $D$ is taut. 

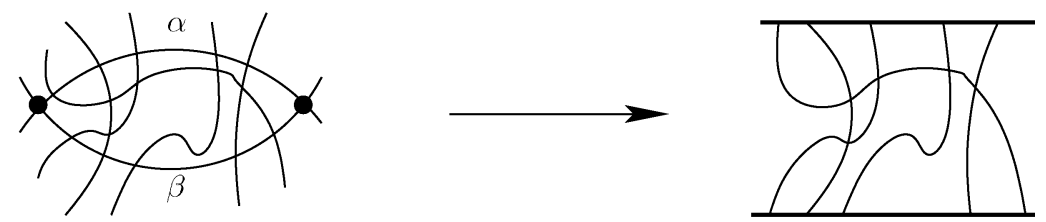

FiguRE 15

Proof. Suppose $D$ is a such a diagram but is not taut. Then let $E$ be a subdiagram as above (i.e., satisfying properties (i)-(iii)) that has no further subdiagram of the same type. Consider the taut braid projection obtained by removing $\alpha$ and $\beta$. If this braid projection has no crossing strands, then they are all parallel. We can then modify the subdiagram $E$ using the move of type II to push each strand past the point $q$ so that the region bounded by $\alpha$ and $\beta$ is empty (Figure 16). Then using the move of type I we can pull $\alpha$ and $\beta$ apart, removing the vertices $p$ and $q$. Since this would decrease the number of interior vertices of $D$ without changing the boundary word $w$, we obtain a contradiction.

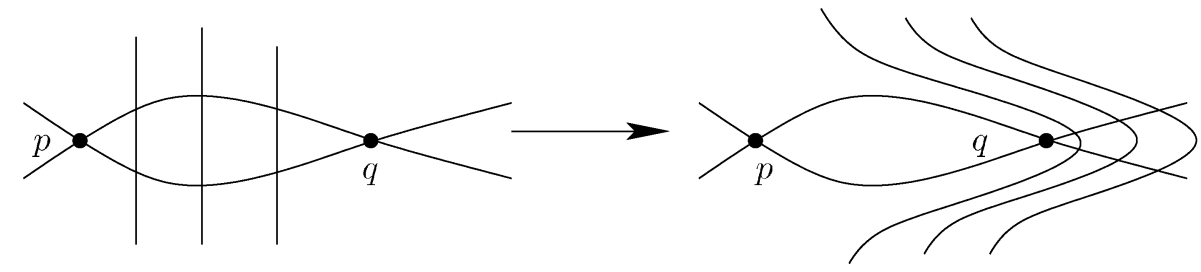

FiguRE 16

We can now assume that the subdiagram $E$ has crossing strands (i.e., there is at least one vertex inside the region bounded by $\alpha$ and $\beta$ ). We claim that the corresponding braid projection must have a triangular region along $\alpha$ (i.e., a triangle at the top). The argument we give here is by induction on the number of strands (see also [11, Lemma 4.5]). There must be at least two strands since there is at least one crossing, and in this case there is obviously a triangle at the top. Assume that any taut braid projection with fewer than $m$ strands has a triangle at the top, and consider the case of $m$ strands. Form a new braid projection by removing the intersection point of the first strand with the top bar, deleting everything to the left and below the first strand, and moving the first strand down parallel to the bottom bar (Figure 17). The new braid is still taut but has fewer strands. If the new braid has no crossings, then the second strand along the top must cross the first strand before crossing any others, which means that the first and second strands in the old braid must bound a triangle at the top. Otherwise, by induction, we know that the new braid has a triangle at the top, hence so does the old one.

Now that we know we must have a triangle at the top, this means we can perform a move of type II on the subdiagram $E$ to decrease the number of interior crossings (Figure 18). Repeating this process, we eventually end up with no interior crossings, so all of the strands are parallel between $\alpha$ and $\beta$. Applying moves II and I, as before, we again obtain a contradiction to the minimality of the number of crossings in $D$. 

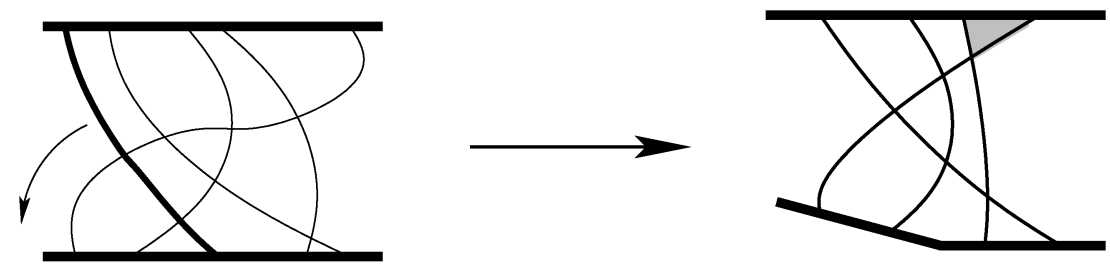

FigURE 17
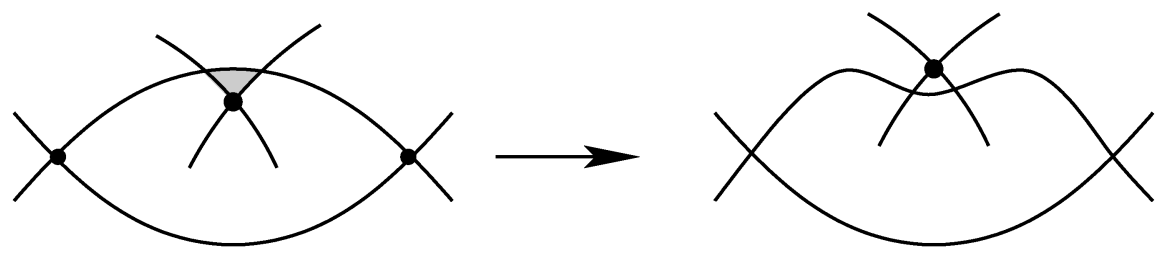

Figure 18

Theorem 3.8. Let $\Gamma$ be a mock reflection system and let $\mathcal{R}$ be the collection of 4-term relations consisting of one relation for each edge set. Let $N$ be the normal subgroup of $F_{2}(V)$ generated by $\mathcal{R}$ and let $w$ be any element in $N$. Then the length $\lambda$ and area $\alpha$ of $w$ satisfy the isoperimetric inequality

$$
\lambda \geq 1+\sqrt{8 \alpha+1} .
$$

Proof. Let $D$ be a chord diagram for $w$ with a minimal number of interior crossings. Then the number of chords in $D$ (that meet the boundary) is precisely $k=\lambda / 2$. By Lemma 3.7, we know that $D$ is taut, so there are at most $\left(\begin{array}{l}k \\ 2\end{array}\right)$ interior crossings. This gives

$$
\alpha \leq \frac{1}{8}\left(\lambda^{2}-2 \lambda\right)
$$

which is equivalent to the desired inequality.

The following example shows that the inequality fails without the no holonomy condition.

Example 3.9. Let $\Gamma$ be the graph shown in Figure 19 with local involutions $j_{a}, j_{b}$, $j_{c}, j_{d}, j_{e}$ as indicated. It is 4 -periodic (but has nontrivial holonomy corresponding to the cycle $a, e, c, d)$. The (reduced) 4-term relations for the group $W(\Gamma)$ are $r_{1}=a e c d, r_{2}=b c a c, r_{3}=a b a b$ and $r_{4}=b d b e$. Figure 20 shows a van Kampen diagram (and dual chord diagram) with boundary word $a b$. Here $\alpha=6$ while $\lambda=2$. In particular, the generators $a$ and $b$ represent the same element in $W(\Gamma)$

The Davis complex. We have seen that any RAMRG has a presentation of the form $W(\Gamma)$ where $\Gamma$ is a mock reflection system. In the converse direction, one would like to say when the group $W(\Gamma)$ defined by a mock reflection system $\Gamma$ is, in fact, a mock reflection group. More precisely, one would like to know when there exists a CAT(0)-cubical complex with an action of $W(\Gamma)$ such that the 1-skeleton of the link of a vertex coincides with the 4-periodic graph $\Gamma$. A partial answer to this question is given in Section 5.10 of [5] (in a setting more general than the right-angled case). As in the case of reflection groups, the idea is to "complete" the 


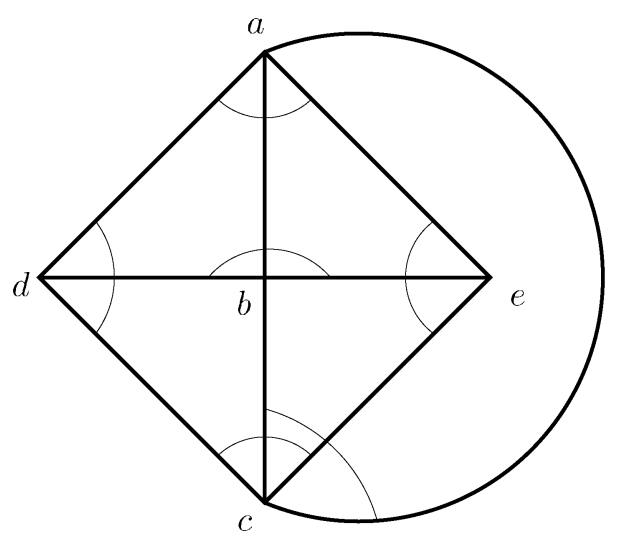

Figure 19

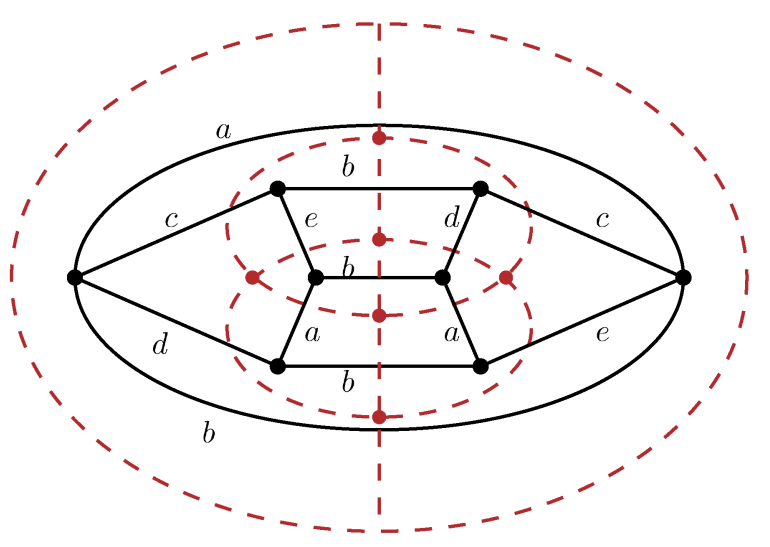

FIGURE 20

Cayley complex of $W(\Gamma)$ by attaching cells so that the links of all vertices become flag complexes. In order for this to work, one needs to be sure that all of the 2-cells in the Cayley complex are embedded. In [5], this is listed as an extra condition, but in the right-angled setting it follows from the isoperimetric inequality we just proved.

Theorem 3.10. Let $\Gamma$ be a right-angled mock reflection system. Then there exists a (unique) $C A T(0)$ cubical complex $\Sigma=\Sigma(\Gamma)$ with an isometric action of $W(\Gamma)$ such that

(i) the action of $W(\Gamma)$ is simply-transitive on $\Sigma^{(0)}$ and the edge-stabilizers are all isomorphic to $\mathbb{Z}_{2}$, and

(ii) the corresponding 4-periodic graph is $\Gamma$.

Proof. We identify $W(\Gamma)$ with the quotient $F_{2}(V) / N$ where $N$ is the normal subgroup generated by the 4-term relations, and we let $X$ denote the corresponding Cayley 2-complex for this presentation. If $r(u, v)=u v u^{\prime} v^{\prime}$ is a relation corresponding to adjacent vertices $u$ and $v$, then any coincidence among the group elements $\overline{1}$, 
$\bar{u}, \overline{u v}$, and $\bar{v}^{\prime}=\overline{u v u^{\prime}}$ in $W(\Gamma)$ would give rise to a word $w$ in $N$ of length $\lambda \leq 3$. By Theorem 3.8 such a $w$ would have a van Kampen diagram with area $\alpha=0$. This means the diagram would have to be a tree, and the only tree whose boundary has length $\leq 3$ is a single edge. But in this case the boundary label would have to be of the form $v v$ for some generator $v$ in $F_{2}(V)$. So there can be no such relator $w$. It follows that the Cayley 2-complex $X$ is a regular square complex. Moreover, the link of every vertex in $X$ coincides with the graph $\Gamma$, and the local involutions on $\Gamma$ are determined by edge labels in $X$ as in Figure 1 of Section 2 ,

To obtain the complex $\Sigma$, one now equivariantly attaches cells to $X$ in such a way as to fill in all of the empty simplices in the links. The difficulty here is that a priori one might have to attach orbifold quotients of cubes instead of cubes. Let $\sigma$ be an empty $(k-1)$-simplex in the link of a vertex (i.e, a complete subgraph) in $X$, and let $X_{\sigma}$ be the subcomplex of $X$ spanned by $\sigma$. That is, $X_{\sigma}$ is the union of the squares spanned by the edges of $\sigma$. Then the no holonomy condition allows one to construct a map $f_{\sigma}: Q^{(2)} \rightarrow X$ from the 2 -skeleton of a $k$ cube to $X$ that is a covering map onto a subcomplex containing $X_{\sigma}$ (see Section 5.7 in [5] for details). In our setting, this covering map will be the restriction of a quotient map for a group $G_{\sigma}$ acting on the full cube $Q$. This allows one to attach (in order of increasing dimensions) the orbifolds $Q / G_{\sigma}$ to $X$ using the maps $f_{\sigma}$. The resulting space $\hat{X}$ has the structure of a piecewise Euclidean orbihedron (see [10]), and since all of the empty simplices in links have been filled in, $\hat{X}$ is nonpositively curved. Since nonpositively curved orbihedra are developable (see [10] and [1]) and $\hat{X}^{(2)}=X$ is simply-connected, $\hat{X}$ must be its own universal orbihedral cover. Since such universal covers have trivial isotropy subgroups, the attached cells must have (a postiori) been cubes. It follows that $\Sigma=\hat{X}$ gives the desired cubical complex $\Sigma$.

The Davis complex $\Sigma(\Gamma)$ can also be described combinatorially by identifying which subsets of $W(\Gamma)$ correspond to the vertex sets of cubes. Let $V$ be the vertex set of $\Gamma$ (i.e., the generators of $W(\Gamma)$ ), and let $L$ denote the collection of subsets $\sigma \subset V$ such that $\sigma$ spans a complete subgraph in $\Gamma$. For each $\sigma$ in $L \cup\{\emptyset\}$, we define an element $w_{\sigma} \in W(\Gamma)$ as follows. We set $w_{\emptyset}=1$ and define the remaining $w_{\sigma}$ 's by induction on $|\sigma|$. Given $\sigma \in L$, pick $v \in \sigma$ and let $w_{\sigma}=v w_{\sigma^{\prime}}$, where $\sigma^{\prime}$ is the (smaller) subset $j_{v}(\sigma-\{v\})$. The 4-periodicity and no holonomy conditions on $\Gamma$ can be used to show that $w_{\sigma}$ is independent of the choice of vertex $v$, hence well defined.

Now for each $\sigma$, we can define subsets $W_{\sigma} \subset W(\Gamma)$ by $W_{\sigma}=\left\{w_{\tau} \mid \tau \subset \sigma\right\}$. Then the cubes in the Davis complex $\Sigma(\Gamma)$ can be identified with the collection of subsets of the form $w W_{\sigma}$, where $w \in W$ and $\sigma \in L \cup\{\emptyset\}$. Note that in the case where $W(\Gamma)$ is an actual reflection group, the subsets $W_{\sigma}$ are precisely the finite parabolic subgroups of $W(\Gamma)$, but in general they need not even be subgroups.

Remark 3.11. One can give a more combinatorial proof of Theorem 3.10 by defining $\Sigma$ to be the geometric realization of the poset of subsets $w W_{\sigma}$, but some form of developability will still be needed. In particular, one needs to show that the putative vertex set $w W_{\sigma}$ for each cube has $2^{|\sigma|}$ distinct elements.

Finite RAMRG's. In this section we characterize all finite right-angled mock reflection groups. 
Theorem 3.12. Let $\Gamma$ be a right-angled mock reflection system. Let $W=W(\Gamma)$ be the associated mock reflection group and let $\Sigma=\Sigma(\Gamma)$ be the Davis complex (of Theorem 3.10). Then the following are equivalent:

(1) $W$ is finite.

(2) $\Gamma$ is a complete graph.

(3) $\Sigma$ is a cube.

In particular, if a RAMRG is finite, then it is a 2-group.

Proof. Suppose $W$ is finite. Let $V$ be the vertices of $\Gamma$ (i.e., the vertices of $\Sigma$ adjacent to $x_{0}$ ), and for $v \in V$ let $s_{v}$ denote the corresponding generator of $W$. Suppose two vertices $u$ and $v$ are not adjacent in $\Gamma$. Then whenever 2-edges in $\Sigma$ with labels $s_{u}$ and $s_{v}$ meet at a vertex, they do not span a square at that vertex. It follows that the sequence $x_{0}, s_{u} \cdot x_{0}, s_{v} s_{u} \cdot x_{0}, s_{u} s_{v} s_{u} \cdot x_{0}, \ldots$ determines a geodesic ray in $\Sigma$. Since $\mathrm{CAT}(0)$ spaces have no closed geodesics, this sequence cannot be periodic, so $s_{u}$ and $s_{v}$ generate an infinite group, contradicting $W$ being finite. It follows that $\Gamma$ is a complete graph.

If $\Gamma$ is a complete graph, the link of every vertex in $\Sigma$ must be a simplex. The only connected cubical complex that has all links isomorphic to an $n$-simplex is the $(n+1)$-cube, so $\Sigma$ must be a cube.

If $\Sigma$ is an $n$-cube, then $G$ has one element for each vertex; hence $W$ is finite with order $2^{n}$. This completes the proof.

Example 3.13. If $\Gamma$ is the complete graph $K_{4}$, there are exactly 4 ways to make it 4-periodic with trivial holonomy. These are shown in Figure 21. The first group is the right-angled Coxeter group $\left(\mathbb{Z}^{2}\right)^{4}$, the second is $\mathbb{Z}_{2} \times D_{4}$, and the last two groups coincide (this can be seen by eliminating the generator $c$ from both presentations). This last group has presentation

$$
\left\langle a, b, d \mid a^{2}=b^{2}=d^{2}=1, a b a b=b d b d,(a d)^{2}=1\right\rangle .
$$

It is a semidirect product of $D_{4}$ and $\mathbb{Z}_{2}$. Notice from Example 3.3 that if the holonomy condition is dropped, $W(\Gamma)$ need not be a 2-group.
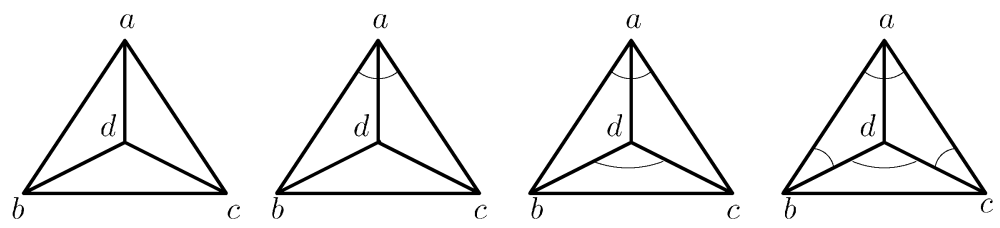

FIGURE 21

\section{Mock Artin groups}

The group $A(\Gamma)$. Let $\Gamma$ be a right-angled mock reflection system with vertex set $V$, and let $F(V)$ denote the free group on $V$. Recall that to define the mock reflection group $W(\Gamma)$ we introduced relations $u v u^{\prime} v^{\prime}=1$ for each trajectory $u, v, u^{\prime}, v^{\prime}, \ldots$ in $\Gamma$ and the involution relations $v^{2}=1$ for each vertex $v \in V$. We would like to define a new group $A(\Gamma)$ without the involution relations, but that still has $W(\Gamma)$ as a quotient group. Thus, $A(\Gamma)$ will be analogous to the Artin group associated to a Coxeter group. 
Given a trajectory $u, v, u^{\prime}, v^{\prime}, \ldots$ in $\Gamma$, let $\tilde{r}(u, v)$ be the relation in $F(V)$ :

$$
\tilde{r}(u, v)=u^{-1} v u^{\prime}\left(v^{\prime}\right)^{-1} \text {. }
$$

Let $N$ be the normal subgroup generated by all $\tilde{r}(u, v)$ for each pair of adjacent vertices $u, v$ in $\Gamma$, and let $A(\Gamma)$ be the quotient group $F(V) / N$. We call $A(\Gamma)$ the (right-angled) mock Artin group associated to $\Gamma$ (or to $W(\Gamma))$. Since each relation $\tilde{r}(u, v)$ is equivalent to the relation $r(u, v)$ when the generators are involutions, there is a canonical surjection $A(\Gamma) \rightarrow W(\Gamma)$.

As in our definition for $W(\Gamma)$ some of the defining relations are redundant for $A(\Gamma)$. In particular, if $u$ and $v$ are adjacent with corresponding trajectory $u, v, u^{\prime}, v^{\prime}, \ldots$, then the relations $\tilde{r}(u, v)$ and $\tilde{r}(v, u)$ are equivalent up to inversion and conjugation. Thus, we will sometimes specify an orientation for each edge of $\Gamma$ and reduce the list of defining relations so there is precisely one relation for each edge.

The Salvetti complex. Given an $n$-dimensional cube $Q$ and an edge $e \subset Q$, let $Q(e)$ denote the $(n-1)$-dimensional subcube obtained by intersecting $Q$ with the hyperplane orthogonal to $E$ passing through the midpoint of $E$. We call $Q(e)$ a hyperplane in $Q$. Any hyperplane in $Q$ determines an equivalence relation on edges by $e \sim e^{\prime} \Leftrightarrow Q(e)=Q\left(e^{\prime}\right)$. More generally, given a cubical complex $X$ (i.e., a regular cell complex all of whose cells are combinatorially isomorphic to cubes), we consider the equivalence relation on edges generated by this relation on each cell. That is, $e$ and $e^{\prime}$ are equivalent if and only if there exists a sequence of edges $e=e_{0}, e_{1}, \ldots, e_{n}=e^{\prime}$ and a sequence of cubes $Q_{1}, \ldots, Q_{n}$ in $X$ such that for $0 \leq i<n, e_{i} \sim e_{i+1}$ in $Q_{i}$.

Given any edge $e$ in $X$, we define the hyperplane $H(e)$ to be the union of all $Q\left(e^{\prime}\right)$ where $e^{\prime} \sim e$ and $Q$ is any cell of $X$ that contains $e^{\prime}$. Given a cube $Q$ in $X$, we say that a hyperplane $H$ is perpendicular to $Q$ if $H=H(e)$ for some edge $e$ in $Q$. Obviously, if a hyperplane is perpendicular to a face of a cube $Q$, then it is also perpendicular to $Q$.

Let $X$ be a cubical cell complex. Let $X^{(0)}$ denote the vertex set of $X$, and let $\mathcal{P}(X)$ denote the poset of cells in $X$. Let $\mathcal{Y}$ be the subset of $X^{(0)} \times \mathcal{P}(X)$ defined by

$$
\mathcal{Y}=\{(x, Q) \mid x \in Q\}
$$

We define a partial order on $\mathcal{Y}$ by $\left(x_{1}, Q_{1}\right) \leq\left(x_{2}, Q_{2}\right)$ if and only if

(1) $Q_{1} \subset Q_{2}$.

(2) $x_{1}$ and $x_{2}$ are on the same side of every hyperplane in $Q_{2}$ that is perpendicular to $Q_{1}$.

That this defines a partial order follows directly from the assumption that $X$ is regular.

The map $\mathcal{Y} \rightarrow \mathcal{P}(X)$ given by $(x, Q) \mapsto Q$ is a map of posets, so there is an induced simplicial map $\pi:\left|\mathcal{Y}^{\prime}\right| \rightarrow X^{\prime}$ where $\left|\mathcal{Y}^{\prime}\right|$ is the geometric realization of $\mathcal{Y}$ and $X^{\prime}$ is the barycentric subdivision of $X$. Given an $n$-cube $Q$ in $X$, the inverse image $\pi^{-1}(Q)$ consists of $n$ copies of $Q$, which can be indexed by the vertices of $Q$. More precisely, if $x$ is a vertex of $Q$, then the point $(x, Q)$ in $\left|\mathcal{Y}^{\prime}\right|$ is the barycenter of the copy of $Q$ corresponding to $x$. In this way, we can give $\left|\mathcal{Y}^{\prime}\right|$ the structure of a cubical cell complex $Y$. We call this cubical cell complex $Y$ the Salvetti complex for $X$. Note that the poset corresponding to $Y$ is $\mathcal{Y}$, so we can identify the element $(x, Q) \in \mathcal{Y}$ with the cell $Q_{x}$ in $Y$. 
Theorem 4.1. Let $X$ be a cubical complex and let $Y$ be the corresponding Salvetti complex, each with its natural piecewise Euclidean metric. Then $Y$ is nonpositively curved if and only if $X$ is nonpositively curved.

Proof. The restriction of the map $\pi: Y \rightarrow X$ to the vertices is a bijection, hence the preimage of the link of a vertex $x$ is the link of the preimage $y=\pi^{-1}(x)$. Let $L$ be the link of the vertex $x$ in $X$, and let $\tilde{L}$ be the link of $y$. By Gromov's condition it suffices to show that $L$ is a flag complex if and only if $\tilde{L}$ is a flag complex.

Let $V$ and $\tilde{V}$ be the vertex sets of $L$ and $\tilde{L}$, respectively. Each $v \in V$ corresponds to an edge $E$ in $X$ with endpoints $x$ and, say, $x^{\prime}$. The preimage of $E$ in $Y$ consists of 2 edges $E_{x}$ and $E_{x^{\prime}}$, which both share the vertex $y=\{x\}_{x}$. Let $v^{+}$and $v^{-}$be the corresponding elements in $\tilde{L}$. Then we have $\tilde{V}=\left\{v^{ \pm} \mid v \in V\right\}$. Similarly, by considering the higher-dimensional cubes at $x$ we see that the simplicial complex $\tilde{L}$ consists precisely of those subsets $\left\{v_{1}^{ \pm}, \ldots, v_{k}^{ \pm}\right\}$such that $\left\{v_{1}, \ldots, v_{k}\right\}$ is a simplex of $L$. (In other words, each simplex in $L$ corresponds to a crosspolytope in $\tilde{L}$.) Hence a subset $\left\{v_{1}^{ \pm}, \ldots, v_{k}^{ \pm}\right\}$of $\tilde{V}$ is pairwise joined by edges in $\tilde{L}$ if and only if $\left\{v_{1}, \ldots, v_{k}\right\}$ is pairwise joined by edges in $L$. It follows that $L$ is a flag complex if and only if $\tilde{L}$ is a flag complex.

A finite $K(\pi, 1)$ for mock Artin groups. Now suppose $\Gamma$ is a mock reflection system with corresponding mock reflection group $W=W(\Gamma)$ and mock Artin group $A=A(\Gamma)$. Let $\Sigma=\Sigma(\Gamma)$ be the Davis complex, and let $Y=Y(\Gamma)$ be the Salvetti complex for $\Sigma$. In this case, since the vertex set of $\Sigma$ can be identified with the group $W$ the cell poset $\mathcal{Y}$ is a subcomplex of $W \times \Sigma$, thus the cells of the geometric realization are of the form $Q_{w}$ for $w \in \Sigma$. In particular, the vertices in $Y$ can also be identified with $W$, and we will often denote the vertex $\{w\}_{w}$ in $Y$ simply by $w$.

The (diagonal) $W$-action on $W \times \Sigma$ restricts to an action on $\mathcal{Y}$ that preserves the partial order. Since the left action of $W$ on itself is free, so is the action on $\mathcal{Y}$. Taking the geometric realization we obtain a free action of $W$ on the Salvetti complex $Y$. We consider the quotient space $Y / W$. For each cell $Q_{w}$ in $Y$, let $\left[Q_{w}\right]$ denote the image in $Y / W$. Let $L$ be the link of 1 in $\Sigma$. Then $L$ is the (unique) flag simplicial complex whose 1-skeleton is the graph $\Gamma$. Each $\sigma \in L$ determines a cube $Q(\sigma)$ in $\Sigma$ that contains the vertex 1. Letting $Q(\emptyset)$ denote the vertex 1 in $Y$, we can define a map $s: L \cup\{\emptyset\} \rightarrow Y / W$ by $s(\sigma)=\left[Q(\sigma)_{1}\right]$. Since any cell $Q_{w}$ can be translated by $w^{-1}$ to a cell of the form $Q_{1}^{\prime}$, we know that $s$ is surjective. On the other hand, the only way two cells $Q(\sigma)_{1}$ and $Q\left(\sigma^{\prime}\right)_{1}$ can be $w$-translates is if $w=1$, in which case $\sigma=\sigma^{\prime}$. It follows that $s$ is a bijection: $Y / W$ consists of a single vertex [1] and a single ( $k$-dimensional) cell $\left[Q(\sigma)_{1}\right]$ for each $(k-1$-dimensional) simplex $\sigma$ in $L$.

Lemma 4.2. The fundamental group $\pi_{1}(Y / W)$ is isomorphic to $A$.

Proof. It suffices to show that the fundamental group of the 2-skeleton of $Y / W$ coincides with the presentation complex for the Artin group $A$. We fix an orientation for each edge in $\Gamma$, and use the presentation for $A$ consisting of the single relation $\tilde{r}(u, v)$ for each such oriented edge $(u, v)$.

Since there is only one vertex in $Y / W$, each vertex in $\Gamma$ determines a loop in $Y / W$. Choose an orientation for this loop as follows. Given the vertex $v$ in $\Gamma$, let $(1, v)$ denote the oriented edge in $\Sigma$ that starts at 1 and ends at $v$. Let $(1, v)_{1}$ be the corresponding orientation for the edge $Q(v)_{1}$ in $Y$, and let $a_{v}$ be the (oriented) 
image loop in $Y / W$. Then $\pi_{1}(Y / W)$ is generated by $\left\{a_{v} \mid v \in V\right\}$, and the relations are determined by the boundary cycles of 2-cells. The map $s$ described above tells us that these 2-cells are in bijection with edges of $\Gamma$. Let $(u, v)$ be an oriented edge in $\Gamma$ and let $Q$ be the corresponding 2-cell in $\Sigma$. The vertices of $Q$ in cyclic order are $1, v, w, u$ where $w=v u^{\prime}=u v^{\prime}\left(\right.$ recall $u^{\prime}=j_{v}(u)$ and $\left.v^{\prime}=j_{u}(v)\right)$. Let $A, B, A^{\prime}, B^{\prime}$ be the oriented edges $(u, 1),(1, v),(v, w),(u, w)$, respectively.

Recall that $Q_{1}$ denotes the copy of $Q$ in $Y$ that corresponds to the vertex 1 . The copies of $A, B, A^{\prime}, B^{\prime}$ that are contained in $Q_{1}$ are $A_{1}, B_{1}, A_{v}^{\prime}$ (since $v$ is on the same side of the hyperplane perpendicular to $A^{\prime}$ as the vertex 1 that indexes $Q_{1}$ ), and $B_{u}^{\prime}$ (see Figure 22). The oriented edge $A_{v}^{\prime}$ is translated to the oriented edge $\left(1, u^{\prime}\right)_{1}$

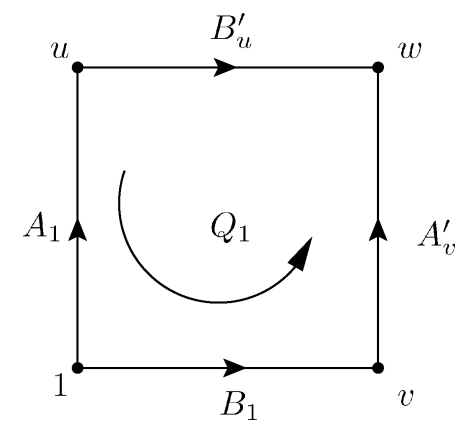

Figure 22. A 2-cell in $Y$

by the element $v$ (since $A_{v}^{\prime}=(v, w)_{v}=\left(v, v u^{\prime}\right)_{v}$ so $\left.v\left(A_{v}^{\prime}\right)=\left(v A^{\prime}\right)_{v v}=\left(1, u^{\prime}\right)_{1}\right)$. Similarly, the oriented edge $B_{u}^{\prime}$ is translated to $\left(1, v^{\prime}\right)_{1}$ by $u$. It follows that the boundary cycle of the 2-cell $\left[Q_{1}\right]$ in $Y / W$ is given by the composition of loops $\left(a_{u}\right)^{-1} a_{v} a_{u^{\prime}}\left(a_{v^{\prime}}\right)^{-1}$. This is precisely the relation $\tilde{r}(u, v)$.

Theorem 4.3. The quotient $Y / W$ is a finite $K(\pi, 1)$ for the mock Artin group $A$.

Proof. Since the $W$ action on $Y$ is free, the universal cover of $Y / W$ coincides with the universal cover of $Y$. By Theorem 4.1 the latter is nonpositively curved, hence contractible by the (generalized) Cartan-Hadamard theorem (see [1, e.g.).

Letting $L$ denote the flag simplicial complex whose 1-skeleton is $\Gamma$, we have the following.

Corollary 4.1. The Euler characteristic of $A(\Gamma)$ is given by $\chi(A(\Gamma))=1-\chi(L)$.

Example 4.4. Let $\Gamma$ be the second triangle graph in Example 3.2. Then $W$ is the dihedral group $D_{4}$, and $A$ is the group

$$
A=\langle a, b, c \mid a b=c a, b a=a c, b c=c b\rangle .
$$

The latter is an HNN-extension of $\mathbb{Z}^{2}$. The generators $b$ and $c$ generate the group $\mathbb{Z}^{2}$, and the third generator $a$ conjugates the corresponding $\mathbb{Z}$ subgroups. In this case, the Davis complex $\Sigma$ is the 3 -cube, the Salvetti complex $Y$ is the 3 -torus with its standard decomposition into eight 3-cubes, and the quotient $Y / W$ is the quotient of the 3-cube shown in Figure 23 (with opposite sides identified so that the labeled arrows match). 


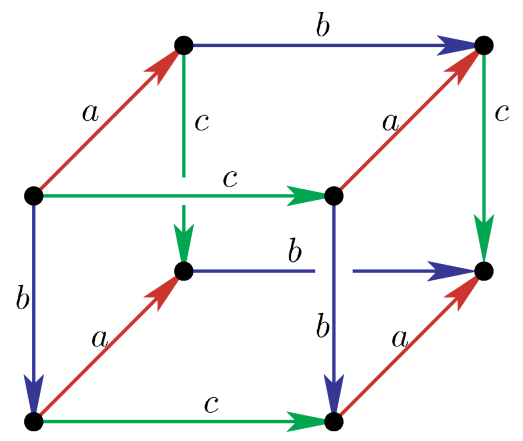

FIGURE 23

\section{REFERENCES}

1. M. Bridson and A. Haefliger. Metric Spaces of Non-positive Curvature. Springer-Verlag, Berlin, Heidelberg, and New York, 1999. MR.1744486 (2000k:53038)

2. R. Charney and M. Davis. Finite $K(\pi, 1)$ 's for Artin Groups. Prospects in topology, Ann. of Math. Stud., 138, Princeton Univ. Press (1995), 110-124. MR.1368655 (97a:57001)

3. M. Davis. Nonpositive curvature and reflection groups. Handbook of Geometric Topology, eds. R. Daverman and R. Sher, North-Holland, Amsterdam (2002), 373-422. MR1886674 (2002m:53061)

4. M. Davis, T. Januszkiewicz, and R. Scott. Nonpositive curvature of blow-ups. Sel. math., New ser. 4 (1998), 491-547. MR1668119 (2001f:53078)

5. M. Davis, T. Januszkiewicz, and R. Scott. Fundamental groups of blow-ups. Adv. Math. 177 (2003), 115-179. MR 1985196 (2004c:57030)

6. P. Etingof, A. Henriques, J. Kamnitzer, and E. Rains. The cohomology ring of the real locus of the moduli space of stable curves of genus 0 with marked points. math.AT/0507514.

7. M. Kapranov. The permutoassociahedron, Mac Lane's coherence theorem and asymptotic zones for the KZ equations. J. Pure and Applied Algebra 85 (1993), 119-142. MR1207505 (94b:52017)

8. M. Kapranov. Chow quotients of Grassmannians. I. Adv. in Sov. Math. 16 (1993), 29-110. MR 1237834 (95g:14053)

9. R. Lyndon and P. Schupp. Combinatorial Group Theory. Springer-Verlag, Berlin, Heidelberg, and New York, 1977. MR0577064 (58:28182)

10. A. Haefliger. Complexes of groups and orbihedra. Group Theory from a Geometrical Viewpoint (A. Ghys, A. Haefliger and A. Verjovsky, eds.), World Scientific Publishing Co., River Edge, NJ, 1991. MR.1170362 (93a:20001)

11. M. Sageev. Ends of group pairs and non-positively curved cube complexes. Proc. London Math. Soc. 71 (1995), no. 3, 585-617. MR1347406 (97a:20062)

Department of Mathematics and Computer Science, Santa Clara University, Santa Clara, California 95053

E-mail address: rscott@math.scu.edu 